\section{A woman in her mid 30s with a dry cough and breathlessness}

A female non-smoker in her thirties was referred to the chest clinic with a 6-month history of a dry cough, breathlessness and general malaise.

Her chest $x$ ray showed diffuse bilateral ground-glass opacity (fig 1). Her autoimmune profile was negative. An HIV test was negative. Lung function tests showed a restrictive pattern with moderately reduced transfer factor.

A high resolution CT (HRCT) showed bilateral ground-glass opacity, more marked on the right, with no evidence of mediastinal or hilar lymphadenopathy (fig 2).

A bronchoscopy showed a normal tracheobronchial tree. Bronchoalveolar lavage contained large numbers of foamy macrophages and increased neutrophils, and microbiogical tests were negative.

A surgical lung biopsy was undertaken (fig 3). Ultrastructural analysis showed giant lamellar structures within the cytoplasm of the alveolar macrophages, and subsequent biochemistry showed sphingomyelinase activity at only $15 \%$ of the normal range, which is consistent with Niemann-Pick disease type B. Abdominal ultrasound showed no organomegaly.

Niemann-Pick disease type B is an inborn error of metabolism where reduced levels of the enzyme sphingomyelinase lead to multisystem disease though with survival into adulthood. ${ }^{1}$ In the lung, this leads to progressive pulmonary infiltrates with significant morbidity.

Classically, there are three types. Type A is the most common disorder and begins shortly after birth with rapid, progressive and fatal neurological involvement and hepatosplenomegaly. Type B is more benign and patients often survive into adulthood, although diagnosis is typically made in childhood due to involvement of other organs such as liver and spleen. In types A and B there is absence or deficiency of the enzyme sphingomyelinase which results in accumulation of sphingomyelin in the target organs. NiemannPick disease type $\mathrm{C}$ is associated with lysosomal accumulation of cholesterol due to a defect in intracellular utilisation of cholesterol, and sphingomyelinase is usually not deficient.

There is no definite treatment for Niemann-Pick disease, although steroids have been used to improve the respiratory symptoms.

Our patient subsequently underwent whole lung lavage and she has benefited symptomatically from this novel treatment.

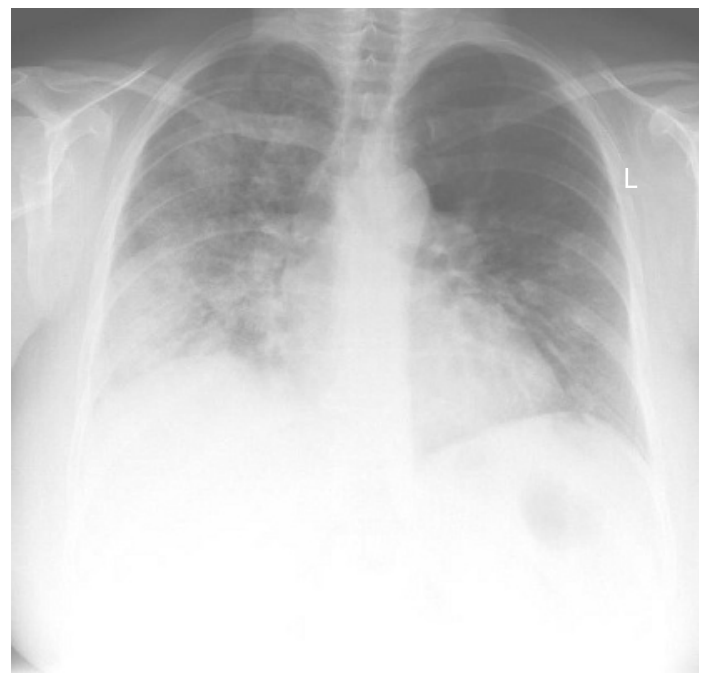

Figure 1 Chest $x$ ray: diffuse bilateral ground-glass opacity.

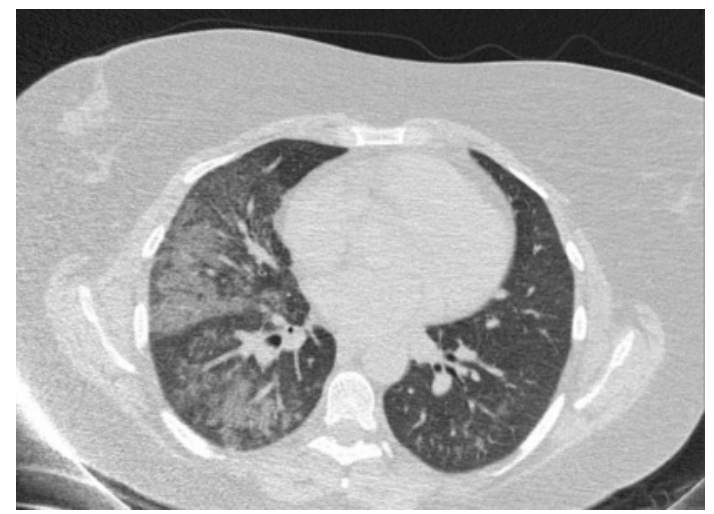

Figure 2 High resolution CT: ground-glass opacity is seen predominantly on the right.

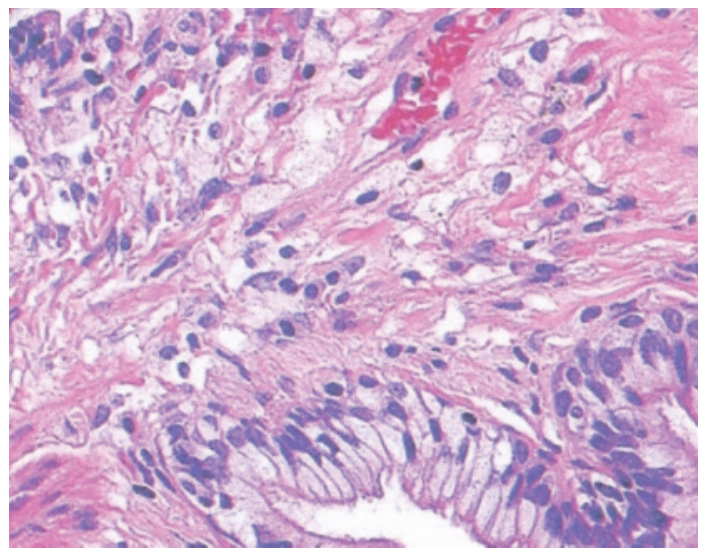

Figure 3 Histology: florid endogenous lipoid pneumonia with a mild interstitial non-specific chronic inflammatory cell infiltrate and mild interstitial fibrosis.

\section{Learning points}

1. Endogenous lipoid pneumonia is a rare disease.

2. In the absence of bronchial obstructive lesions and relevant drug history, inborn errors of metabolism should be suspected particularly in the context of recurrent or persistent radiological changes.

3. Whole lung lavage is a novel therapy which leads to symptomatic improvement in endogenous lipoid pneumonia due to Niemann-Pick disease.

Haematopoietic stem cell transplantation and gene therapy may alter the course of lysosomal storage diseases in the future.

\section{H K Ramadan, ${ }^{1}$ C I Mackinlay, ${ }^{2}$ A G Nicholson ${ }^{3}$}

${ }^{1}$ Respiratory Department, Royal Hampshire County Hospital, Winchester, UK;

${ }^{2}$ Respiratory Department, Great Western Hospital, Swindon, UK; ${ }^{3}$ Histopathology Department, Royal Brompton Hospital, London, UK

Correspondence to: Dr H K Ramadan, Respiratory Department, Royal Hampshire County Hospital, Winchester SO22 5DG, UK; hkhk00@hotmail.com

Competing interests: None.

Patient consent: The details of this case have been anonymised.

Thorax 2009;64:644. doi:10.1136/thx.2008.107243

\section{REFERENCE}

1. Schuchman EH, Desnick RJ. Niemann-Pick disease types A and B: acid sphingomyelinase deficiencies. In: Scriver CR, Beaudet AL, Sly WS, Valle D, eds. The metabolic and molecular base of inherited disease. 7th edn. New York: McGraw Hill, 1995:2601-38. 